\title{
VALORAÇÃO AMBIENTAL DE ÁREAS DE PRESERVAÇÃO PERMANENTE DA MICROBACIA DO RIBEIRÃO SÃO BARTOLOMEU NO MUNICÍPIO DE VIÇOSA, MG ${ }^{1}$
}

\author{
Ana Dantas Mendez de Mattos², Laércio Antônio Gonçalves Jacovine ${ }^{3}$, Sebastião Renato Valverde ${ }^{3}$, \\ Agostinho Lopes de Souza ${ }^{3}$, Márcio Lopes da Silva ${ }^{3}$ e João Estáquio de Lima ${ }^{4}$
}

\begin{abstract}
RESUMO - O objetivo deste trabalho foi estimar o valor monetário das áreas de preservação permanente da microbacia do ribeirão São Bartolomeu, localizada no Município de Viçosa, Minas Gerais. Utilizou-se o Método de Valoração Contingente para estimar a verdadeira disposição a pagar (DAPv) da população de Viçosa pela recuperação ou preservação dessas áreas. A DAPv mensal foi estimada em $\mathrm{R} \$ 27,98$ por domicílio, que resulta, considerando-se a cidade de Viçosa, o montante anual de $\mathrm{R} \$ 3.863 .926,08$ para o bem ambiental em questão ou R $\$ 3.616,52 /$ ha. ano para a recuperação e, ou, preservação dessas áreas.
\end{abstract}

Palavras-chave: Valoração contingente, áreas de preservação permanente, e disposição a pagar.

\section{ENVIRONMENTAL VALUATION OF PERMANENT PRESERVATION AREAS IN SÃO BARTOLOMEU WATERSHED, VIÇOSA, MINAS GERAIS}

\begin{abstract}
The objective of this work was to estimate an economic value for the Permanent Preservation Areas in São Bartolomeu watershed, located in Viçosa, MG. The Contingent Valuation Method was used to estimate the true willingness-to-pay (WTP) of the city of Viçosa residents for the restoration or preservation of these areas. The monthly true WTP was estimated at $R \$ 27.98$ per residence, which results in an approximated total value of $R \$ 3.863 .926 .08$ or $R \$ 3.616 .52 /$ ha.year for the restoration or preservation of the areas.
\end{abstract}

Keywords: Contingent valuation, areas of permanent preservation and willingness-to-pay.

\section{INTRODUÇÃO}

Há tempos, acreditava-se que os recursos ambientais eram abundantes e que nunca iriam se exaurir. Assim, não se via necessidade de valorá-los economicamente. O valor atribuído ao meio ambiente era zero, ou seja, era considerado como bens gratuitos que não entravam na contabilidade econômica, apesar de ser usado na produção de bens e serviços (BENAKOUCHE e CRUZ, 1994). Com o passar do tempo, entretanto, a perspectiva de que esses recursos poderiam se tornar escassos e até se extinguirem fez que surgisse a idéia de desenvolvimento sustentável.

Diante desse contexto, a fim de evitar o risco do uso excessivoe sua completa degradação, ficou clara a necessidade de se atribuir valor positivo aos recursos ambientais. Por fim, a valoraçãoeconômica ambiental se tornou imprescindível ao desenvolvimento das bases econômicas para o estabelecimento de políticas ambientais (MAIA, 2002).

\footnotetext{
${ }^{1}$ Recebido em 23.08.2006 e aceito para publicação em 05.12.2006.

${ }^{2}$ Engenheira Florestal. E-mail: <anadmattos@yahoo.com.br>.

${ }^{3}$ Departamento de Engenharia Florestal da Universidade Federal de Viçosa. E-mail:<jacovine@ufv.br $>$.

${ }^{4}$ Departamento de Economia Rural da Universidade Federal de Viçosa. E-mail: <jelima@ ufv.br>.
} 
As florestas, assim como outros recursos ambientais, vêm sendo degradados no Brasil em ritmo acelerado e, em algumas regiões, como a de ocorrência da mata atlântica, restam apenas áreas degradadas e com pequenos fragmentos florestais. Diante dessa questão, é necessário que sejam dirigidos esforços para valoração dos recursos florestais, de forma a garantir a sua proteção por meio de incentivos econômicos.

Entre os tipos de florestas de proteção, as Áreas de Preservação Permanente (APP) merecem atenção especial, em razão da sua importância na prestação de serviços ambientais para toda a sociedade. As APPs são áreas protegidas pelo Código Florestal (BRASIL, 1965), cobertas ou não por vegetação nativa com a função ambiental de preservar os recursos hídricos, a paisagem, a estabilidade geológica, a biodiversidade, o fluxo gênico da fauna e da flora, proteger o solo e assegurar o bem-estar das populações humanas. Essas áreas se localizam nos topos de morros, encostas, margens de rios etc., e nelas os recursos naturais não podem ser explorados.

Calabria (2004), ao estudar a aplicabilidade da legislação florestal nas propriedades rurais da região da Zona da Mata de Minas Gerais, concluiu que os produtores rurais não a estão cumprindo no tocante às APPs e de reserva legal. O problema se agrava na região porque, pela alta incidência de cursos d'água e topografia acidentada, o que faz que grande extensão das propriedades rurais seja considerada de preservação permanente. A autora concluiu que as condições sociais, econômicas e ambientais das pequenas propriedades mostraram-se limitantes para a aplicação das normas legais.

Franco (2000) relatou que outro problema é a estrutura fundiária da região, que é composta, em sua maioria, por minifúndios. Os pequenos agricultores se deparam com uma situação adversa dentro de suas propriedades, pois o porcentual obrigatório da reserva legal $(20 \%)$ soma-se à alta porcentagem de APPs, como matas ciliares, encostas, topos de morro e entorno de nascentes, ainda que em algumas situações específicas a APP possa ser computada como reserva legal. Essas áreas são de grande importância para os pequenos agricultores de alimentos e foram ocupadas com culturas anuais de feijão e milho, nas margens de cursos d'água; e pastagens e café, nas encostas e topos de morro.
As razões para descumprimento da lei são muitas, mas acredita-se que uma delas é a idéia de que as áreas com floresta nativa são improdutivas e sem valor econômico, tornando qualquer outra forma de uso mais rentável. Daí o risco de a fronteira agrícola continuar em expansão enquanto a floresta não for atraente econômica e socialmente para os diferentes agentes do meio rural.

Os proprietários rurais que respeitam a lei deveriam receber compensação financeira pela preservação de suas APPs. AAPP gera serviços ambientais para todos, inclusive para os que não cumprem a lei. Entre esses serviços, pode-se citar: a proteção dos recursos hídricos e do solo, a regulação climática e a qualidade do ar, a biodiversidade e o sequiestro de carbono. Este último já com perspectiva de ganho econômico, porém ainda há muita indefinição, sobretudo quando se trata de vegetação nativa e pequenas áreas.

Dessa forma, acredita-se que há a necessidade de alguma forma de benefício econômico para que esses proprietários rurais mantenham a vegetação nativa nas áreas determinadas pela legislação. Como os benefícios gerados pela manutenção das APPs são para toda a sociedade, uma solução seria ela pagar para sua proteção.

Para que haja o pagamento para proteção dos recursos florestais e ambientais, é necessário que seja estimado o valor do bem em questão para a sociedade, de forma a subsidiar o estabelecimento de políticas de proteção ambiental. Por isso, o objetivo deste trabalho foi determinar o valor monetário das APPs da Microbacia do ribeirão São Bartolomeu, com base no valor aceito pela população de Viçosa, MG.

\section{MATERIAL E MÉTODOS}

\subsection{Caracterização da área de estudo}

A microbacia hidrográfica do ribeirão São Bartolomeu está totalmente inserida no Município de Viçosa, na região da Zona da Mata de Minas Gerais, e se localiza entre os paralelos $20^{\circ} 44^{\prime}$ e $20^{\circ} 50^{\prime}$ latitude sul e entre os meridianos $42^{\circ} 51^{\prime}$ e $42^{\circ} 53^{\prime}$ ' longitude oeste de Greenwich (ARRUDA, 1997).

O relevo é acidentado, com grande incidência de recursos hídricos e altitudes variando de 600 a 1.200 m. Segundo Ferreira e Dias (2004), o ribeirão São Bartolomeu é responsável por parte do abastecimento de água da cidade de Viçosa e da sua Universidade 
Federal. Toda a região se compõe de pequenas propriedades rurais. Cerca de $90 \%$ dos estabelecimentos possuem área com até 100 hectares, o que faz que a região tenha a estrutura fundiária menos concentrada de Minas Gerais (FRANCO et al., 2002).

Por causa das características físicas da região, a microbacia possui grande extensão de áreas consideradas de preservação permanente. Segundo Franco (2000), dos 5.603,39 ha que a microbacia ocupa, as APPs representam 1.068,41 ha $(19,06 \%)$ da microbacia. Desse total de áreas consideradas APPs, 54,14\% estão ocupadas com pastagem, agricultura e reflorestamento. O total de áreas com uso indevido é de 578,53 há, que correspondem a $10,32 \%$ da área total da microbacia.

\subsection{Fonte e coleta dos dados}

Os dados utilizados neste trabalho foram procedentes de fonte primária, coletada por meio de entrevistas feitas nos meses de outubro e novembro de 2005, na zona urbana do Município de Viçosa, Minas Gerais. O levantamento foi realizado por meio de questionários (survey) aplicados a uma amostra da população da área de estudo, em três pontos de grande fluxo de pessoas na cidade. A amostra foi aleatória, a fim de possibilitar a avaliação da influência das variáveis. A única restrição da escolha foi o fato de a pessoa ter mais de 18 anos, pois acreditou-se que, geralmente, maiores de idade podem responder melhor pelas suas escolhas, e, como a técnica se baseia na disposição a pagar, é necessário que o entrevistado tenha alcançado a idade que lhe permite trabalhar e auferir renda.

O tamanho da amostra foi estimado pela metodologia proposta por Gil (1995) para populações finitas (abaixo de 100.000 observações):

$$
n=\frac{\delta^{2} \cdot p \cdot q \cdot N}{e^{2}(N-1)+\delta^{2} \cdot p \cdot q}
$$

em que $n$ é o tamanho da amostra; $\delta^{2}$, estimativa do nível de confiança escolhido, expresso em número de desvios-padrão; $p$, probabilidade de o fenômeno ocorrer; $q$, probabilidade complementar; $N$, tamanho da população; $e^{2}$, erro máximo permitido.

Para determinação da amostra da pesquisa, adotaramse dois desvios-padrão como nível de confiança. Para a probabilidade de ocorrência do evento " $p$ ", adotou- se o valor máximo 0,5 , já que essa estimativa não foi estabelecida previamente; conseqüentemente, " $q$ " é igual a 0,5. O tamanho da população do Município de Viçosa “ $N$ " utilizado foi de 71.624 habitantes, conforme IBGE (2004). O erro máximo permitido adotado foi $6 \%$. A equação (1) resultou em uma amostra de 277 indivíduos, que foi arredonda para 280 .

\subsection{Modelos utilizados}

Para determinar o valor monetário das APPs da Microbacia do Ribeirão São Bartolomeu, por meio do valor aceito pela população urbana de Viçosa, foi utilizado o método de valoração contingente (MVC), em que se medem as preferências individuais a partir da disposição a pagar (DAP), nesse caso pela recuperação ou preservação das APPs da microbacia. Para tal, baseouse em Seroa da Motta (1998) e Silva (2003).

Foi criado um mercado hipotético, no qual as pessoas pagariam uma taxa adicional, na conta de água, para ser direcionada à recuperação ou preservação de APPs. Aliado a isso, procurou-se também avaliar a percepção ambiental da população urbana em relação à legislação florestal e aos serviços ambientais prestados pelas APPs da microbacia.

A técnica de eliciação utilizada para determinar a DAP foi uma adaptação do método referendum com jogos de leilão. Foram oferecidos diferentes lances iniciais, conforme critérios preestabelecidos. Caso o valor inicial proposto fosse aceito, aumentava-se o lance até a pessoa dizer não; caso contrário, diminuíase o valor até obter uma resposta positiva.

Foi utilizado o modelo logit para identificar as variáveis que influenciavam a probabilidade de os indivíduos aceitarem a DAP proposta pela recuperação ou preservação das APPs. Esse modelo se baseia na utilização da Função de Distribuição Acumulada Logística (FDAL) e é usado para estimar a DAPverdadeira (DAP $)$. Tem-se a seguinte expressão para a FDAL:

$$
P_{i}=P(Y=1)=f\left(\beta X_{i}\right)=\frac{1}{1+e^{-\beta X}}
$$

em que $P_{i}$ corresponde à probabilidade de o indivíduo i tomar a decisão $\left(Y_{i}=1\right)$, isto é, aceitar o valor proposto; $\mathrm{b} X_{i}$ é um índice que representa as características desse indivíduo; i, cada observação da amostra utilizada; e " $e$ ", a base de logaritmos neperianos.

R. Árvore, Viçosa-MG, v.31, n.2, p.347-353, 2007 
A partir dessa equação, tem-se o seguinte modelo econométrico:

$L_{i}=\beta_{1}+\beta_{2} D A P_{p i}+\beta_{3} R F_{i}+\beta_{4} S E X_{i}+\beta_{5} I D_{i}+\beta_{6}$ GINST1 $_{i}$ $+\beta_{7}$ GINST2 $_{i}+\beta_{8}$ GINST3 $_{i}+\beta_{9}$ BECO $_{i}+\beta_{10} C L_{i}+\beta_{11} C D_{i}$ $+\beta_{12} A V_{i}+\beta_{13} M P E_{i}+\varepsilon_{i}$

em que $\beta_{i}(i=1$ a 13) são os parâmetros a serem estimados; $L_{i}$, o logit do indivíduo; $D A P_{p}$, o valor da Disposição a Pagar proposto ao entrevistado; $R F_{i}$, renda familiar mensal, em reais; $\mathrm{SEX}_{\mathrm{i}}$, uma variável dummy: 1 - mulher,

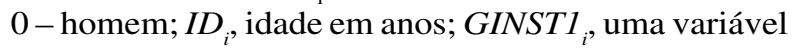
dummy representativa dos indivíduos que possuem ensino fundamental (completo ou não). Será 1 se possuir e 0 , caso contrário; GINST2 , uma variável dummy representativa dos indivíduos que possuem ensino médio (completo ou não), sendo 1 se possuir e 0 , caso contrário; GINST3 i, uma variável dummy representativa dos indivíduos que possuem ensino superior (completo ou não). Será 1 se possuir e 0 , caso contrário; $B E C O_{i}$, dummy assumindo o valor 1 se o entrevistado acha que os proprietários rurais devem receber algum benefício econômico para recuperarem ou preservarem as APPs e 0 , caso contrário; $C L_{i}$, dummy que assume o valor 1 se o entrevistado afirma ter conhecimento da legislação florestal e 0 , caso contrário; $C D_{i}$, dummy que assume o valor 1 se o entrevistado diz ter conhecimento dos danos gerados pelo desmatamento das APPs e 0 , caso contrário; $A V_{i}$, dummy assumindo o valor 1 caso o entrevistado ache que o desmatamento das APPs afeta a sua vida pessoal e 0 , caso contrário; $M P E_{i}$, dummy que assume o valor 1 caso o entrevistado ache que os proprietários rurais não recuperam ou preservam as APPs por motivos econômicos e 0 , caso contrário; e $\varepsilon$, o erro aleatório.

\section{RESULTADOS E DISCUSSÃO}

Foram abordadas 468 pessoas, sendo que, destas, 280 se dispuseram a participar da pesquisa. As outras 188 se recusaram a participar, atingindo um alto índice de recusa (40\%). Isso se deve, principalmente, à forma de abordagem da pesquisa, com entrevistas feitas na rua, realizadas geralmente em horário comercial, com escolha aleatória da pessoa a ser entrevistada. Muitos não estavam dispostos a colaborar, declarando estar com pressa.

\subsection{Perfil da amostra}

Dos 280 entrevistados, 45,4\% (123) foram do sexo feminino e 54,6\% (153), do sexo masculino. Quanto à instrução, pode-se verificar que mais de $60 \%$ da amostra possuía no mínimo o ensino médio completo. $\mathrm{O}$ alto nível de instrução pode ser explicado pelo fato de a cidade possuir aproximadamente $10 \%$ da sua população de estudantes universitários e ter como principal atividade econômica a educação.

A grande maioria dos domicílio (mais de 70\%) tinha renda mensal até cinco salários mínimos, e pouco mais de 5\% recebiam mais de 10 salários mínimos. A população da amostra foi predominantemente jovem, visto que mais de $50 \%$ da amostra tem até 35 anos.

\subsection{Disposição a Pagar}

Apenas 55\% das pessoas se mostraram dispostas a contribuir com alguma quantia para a recuperação e, ou, preservação das APPs da Microbacia do Ribeirão São Bartolomeu, Viçosa, MG. Brugnaro (2000) obteve resultado semelhante: cerca de $42 \%$ dos entrevistados estavam dispostos a pagar alguma quantia para a recuperação da mata ciliar da Bacia do Rio Corumbataí, SP.

Quando se trata de outros bens ambientais de que a população amostrada usufrui diretamente, como parques, é comum obter maior número de DAP positiva. Martins (2002) obteve o índice de 75,8\% de DAP pelos recursos ambientais da praia de Jericoacoara, Ceará. Silva (2003), ao avaliar a DAP para a manutenção e conservação do Parque Ambiental "Chico Mendes", obteve o índice de $68 \%$ de respostas positivas ao pagamento de alguma quantia.

Em relação à justificativa para o não-pagamento de nenhuma quantia, os motivos mais citados foram: "já paga muitos impostos e taxas" (30\%), "o problema é do governo" (27\%) e "não confia no uso dos recursos" (16\%). Esses motivos captam atitudes de protesto e indicam o viés do subdesenvolvimento por causa das altas taxas tributárias impostas pelo governo brasileiro e o descrédito no uso dos recursos quando repassados para os órgãos públicos. As pessoas consideram que o governo deveria remanejar o repasse dos recursos atuais e não aumentar mais a carga tributária. O motivo "minha renda não permite" também indica um viés do subdesenvolvimento, demonstrando a influência do baixo nível de renda na DAP. O motivo "o problema é dos produtores rurais" também indica uma manifestação de protesto, evidenciando que as pessoas costumam se eximir de responsabilidades ambientais. 
Ainda existem aqueles que não vêem importância no assunto. Esse motivo capta aquela parcela da população que acha que a implementação do projeto não irá alterar sua utilidade. Isso revela a falta de conhecimento real dos serviços ambientais promovidos pela preservação do meio ambiente, pois, apesar de o alto grau de escolaridade da amostra, foi considerável o índice de pessoas que não se importavam muito com a questão. Vê-se que ainda não é dada a devida importância ao assunto meio ambiente no ensino escolar.

Apenas para a estimativa paramétrica, foram descartados 13 questionários, já que apresentavam valores muito discrepantes (outliers) em relação ao resto da amostra e prejudicavam os ajustes. Dessa forma, restaram 267 questionários com 1.002 observações. Feito tal procedimento, estimou-se o modelo (03) por meio do software EVIEWS 4.1, cujos resultados se encontram no Quadro 1.

O modelo apresentou seis variáveis estatisticamente significativas ( $D A P, R F, S E X, I D, B E C O$ e $C L)$, sendo três delas significativas a $1 \%(S E X, I D$ e $B E C O)$. A $D A P_{p}$ foi incluída no modelo, apesar de não ter sido significativa a $10 \%$, já que é aceita a significância estatística de até $15 \%$. O porcentual de previsões corretas do modelo foi de $85,03 \%$.

O modelo indica que a probabilidade média de um indivíduo apresentar disposição a pagar positiva é de $15,20 \%$, obtida pela equação (02). Em estudos de valoração de bens ambientais, Brugnaro (2000) obteve probabilidade de pagamento de $82 \%$, já Silva (2003), $24,12 \%$.

Não se optou por reestimar o modelo sem as variáveis não-significativas, pois a retirada dessas afetava consideravelmente o ajuste. Na simulação do modelo reduzido, a DAP proposta não aparecia como variável significativa.

O sinal do coeficiente da $D A P_{p}$ foi negativo, o que confirma a teoria econômica de que, quanto maior o valor oferecido ao entrevistado, menor a probabilidade dele em aceitar pagar. O efeito marginal da variável $D A P_{p}$ indica que, mantendo-se as outras variáveis constantes, o aumento em uma unidade monetária no valor oferecido provocará diminuição na probabilidade de pagamento em 0,008 ou 0,8 ponto porcentual.

O efeito marginal da variável $R F$ (Renda Familiar) foi pequeno, já que o seu aumento em uma unidade monetária eleva em apenas 0,004 ponto porcentual a probabilidade de o indivíduo ter uma disposição a pagar positiva. Esperava-se uma relação direta entre $D A P_{v}$ e a renda, posto que, conforme explicaram Pearce e Turner (1990), as classes de renda mais alta são as que demandam melhorias ambientais, já que tiveram as suas necessidades básicas e materiais satisfeitas. Portanto, almejam melhorar sua qualidade de vida, aumentando seu nível de bem-estar, o que muitas vezes é alcançado por meio de melhorias ambientais.

Quadro 1 - Resultados do modelo logit para estimação da $\mathrm{DAP}_{\mathrm{v}}$ pela recuperação ou, preservação das APPs da microbacia do ribeirão São Bartolomeu, Viçosa, MG, 2005

Table 1 -Results of the logit model to estimate true willingness-to-pay for the PPA's restoration or preservation of São Bartolomeu watershed, Viçosa, MG, 2005

\begin{tabular}{|c|c|c|c|c|c|}
\hline Variável & Coeficiente & Erro padrão & Teste-z & Valor-p & Efeito marginal \\
\hline Intercepto & $-1,947393$ & 0,793500 & $-2,454181$ & $0,0141 * *$ & - \\
\hline $\mathrm{DAP}_{\mathrm{p}}$ & $-0,060863$ & 0,040600 & $-1,499104$ & $0,1338 *$ & $-0,007844$ \\
\hline RENDA & 0,000306 & 0,000136 & 2,250625 & $0,0244 * *$ & 0,000039 \\
\hline SEXO & 0,682457 & 0,197851 & 3,449347 & $0,0006 * * *$ & 0,033908 \\
\hline IDADE & $-0,029150$ & 0,009036 & $-3,226073$ & $0,0013 * * *$ & $-0,003757$ \\
\hline GINST1 & 0,135280 & 0,620769 & 0,217923 & $0,8275^{\mathrm{NS}}$ & - \\
\hline GINST2 & $-0,003841$ & 0,591668 & $-0,006491$ & $0,9948 \mathrm{NS}$ & - \\
\hline GINST3 & 0,142337 & 0,601464 & 0,236650 & 0,8129 NS & - \\
\hline BECO & 1,080975 & 0,219230 & 4,930769 & $0,0000 * * *$ & 0,032037 \\
\hline CL & 0,487996 & 0,220307 & 2,215076 & $0,0268 * *$ & 0,050636 \\
\hline $\mathrm{CD}$ & $-0,065422$ & 0,317380 & $-0,206133$ & $0,8367 \mathrm{NS}$ & - \\
\hline AV & $-0,035000$ & 0,377961 & $-0,092602$ & $0,9262 \mathrm{NS}$ & - \\
\hline MPE & $-0,239316$ & 0,197125 & $-1,214032$ & $0,2247 \mathrm{NS}$ & - \\
\hline
\end{tabular}

Fonte: Resultados da pesquisa.

Obs: * coeficiente significativo a 10\%, ** coeficiente significativo a 5\%, *** coeficiente significativo a $1 \%$ e NS parâmetro não-significativo. 
A variável $S E X$ (Sexo) apresentou coeficiente positivo, o que indica uma maior probabilidade das pessoas do sexo feminino terem disposição a pagar positiva. $\mathrm{O}$ efeito marginal indica um acréscimo de aproximadamente 3,4 pontos percentuais sobre a probabilidade de o indivíduo aceitar pagar, caso seja mulher. Silva (2003) obteve resultado contrário. O fato de o entrevistador ser do sexo feminino ou masculino pode ter influenciado os resultados.

A variável ID (Idade) apresentou coeficiente negativo. O efeito marginal negativo indica que a elevação em um ano de vida reduz a probabilidade de pagamento em aproximadamente 0,38 ponto porcentual. $O$ resultado aponta que as pessoas mais jovens apresentam maior probabilidade de pagar pelo bem em questão. A causa disso pode estar relacionada com o avanço da educação ambiental no ensino formal nas escolas e com o fato de os jovens serem mais conscientes dos problemas ambientais.

Quanto à variável dummy BECO (Benefício Econômico), verificou-se efeito positivo, conforme esperado. Em outras palavras, o fato de achar que os produtores rurais devem receber algum tipo de benefício econômico (BECO) para recuperar ou preservar suas APPs aumenta em 3,2 pontos porcentuais a probabilidade da pessoa em aceitar o pagamento.

A variável dummy CL (Conhecimento da Legislação) também possui efeito marginal positivo. Logo, quando as pessoas demonstram ter conhecimento da legislação que trata das áreas de preservação permanente, a probabilidade de se obter uma disposição a pagar positiva aumenta em 5,06 pontos porcentuais.

Em relação às variáveis que não se mostraram estatisticamente significativas, não se pode inferir sobre seus efeitos marginais, pois a probabilidade de seus parâmetros estimados serem zero é alta.

Nenhuma das variáveis dummies que representaram o fator do grau de instrução (GINST1, GINST2 e GINST3) influenciou estatisticamente a decisão do entrevistado em aceitar o pagamento proposto, indo contra as expectativas iniciais. Isto é, a disposição a pagar das pessoas pela recuperação ou preservação das APPs independe do nível de instrução. Assim como neste trabalho, Brugnaro (2000) e Silva (2003) também não acharam relação estatística significativa entre a escolaridade e a disposição a pagar por um bem ambiental.
As variáveis que procuravam avaliar a percepção ambiental das pessoas ( $C D$ e $A V$ ) também não se mostraram estatisticamente significativas. É possível que o conhecimento dos danos do desmatamento das APPs não tenha afetado a decisão do pagamento do indivíduo porque, apesar de nem todos demonstrarem conhecê-los realmente, é consenso que a retirada da vegetação nativa acarreta conseqüências ruins. $\mathrm{Na}$ mesma linha, todos concordaram que os danos ambientais são preocupantes e podem comprometer a qualidade de vida, afetando a vida pessoal.

A variável que avaliava se os proprietários rurais desmatavam por motivos econômicos (MPE) também foi estatisticamente não-significativa. Portanto, as justificativas econômicas dos proprietários rurais para desmatar as APPs não fazem diferença na decisão de aceitar ou não o pagamento de um valor pela recuperação ou preservação das APPs. Talvez isso indique que as pessoas não consideram essa justificativa aceitável, visto que toda a sociedade deve respeitar a lei, independentemente de qualquer coisa.

A partirda estimaçãodo modelo logit, pode-se determinar a DAP mensal máxima para a população urbana de Viçosa. Como proposto, segue-se a abordagem de Hanemann, substituindo-se os coeficientes das variáveis estatisticamente significativas do Quadro 1 na equação (4). $\mathrm{ADAP}_{\mathrm{v}}$ obtida foi de $\mathrm{R} \$ 27,98$ por mês.

Martins (2002) concluiu que cada visitante de Jericoacoara, CE, está disposto a pagar em média $\mathrm{R} \$ 50,01$ por visita, enquanto Silva (2003) achou o valor $\mathrm{R} \$ 7,60$ para manutenção e conservação do Parque Ambiental Chico Mendes, AC.

Considerando as 11.508 ligações domiciliares de água do Serviço Autônomo de Água e Esgoto (SAAE) de Viçosa, MG, esse número resulta no valor anual de R\$3.863.926,08 para o bem ambiental em questão. Sabendo que as APPs da Microbacia do Ribeirão São Bartolomeu correspondem a uma área de 1.068,41 ha, obtém-se o valor R \$3.616,52 por hectare ao ano para a recuperação ou preservação dessas áreas.

\section{CONCLUSÃO}

Diante dos resultados, pode-se concluir que:

- A população demonstrou pouco conhecimento em relação ao Código Florestal e às funções ambientais das APPs, mas se mostrou consciente da importância da preservação e a favor da implantação de projetos de recuperação dessas áreas. 
- A maioria dos viçosenses acha que os proprietários rurais devem receber algum tipo de benefício econômico para recuperar e ou preservar suas APPs e espera que o governo pague por isso.

- A DAP média por domicílio foi de $\mathrm{R} \$ 27,98$ por mês. Pode-se dizer que é um valor considerável, ainda mais se levando em conta as restrições econômicas da região.

- Os resultados podem contribuir para o estabelecimento de políticas alternativas visando à recuperação e, ou, preservação dessas áreas.

- Os valores revelaram a importância das APPs para as pessoas e como a preservação dessas áreas afeta o seu bem-estar, isto é, sua qualidade de vida. Os resultados deste trabalho revelaram um possível mercado para esse bem ambiental e fornecem uma idéia de como seria o comportamento dos consumidores caso ele existisse.

\section{REFERÊNCIAS}

ARRUDA, P. R. R. Uma contribuição ao estudo ambiental da Bacia Hidrográfica do Ribeirão São Bartolomeu, Viçosa, Minas Gerais. 1997. 108f. Dissertação (Mestrado em Ciência Florestal) - Universidade Federal de Viçosa, Viçosa, MG, 1997.

BENAKOUCHE, R.; CRUZ, R. S. Avaliação monetária do meio ambiente. São Paulo: Makron Books do Brasil, 1994. 198p.

BRASIL. Lei 4.771 de 15 de setembro de 1965 Instituiu o Novo Código Florestal (com alterações introduzidas pela Lei 7.803, de 18 de julho de 1989 que Altera a redação da Lei 4.771 de 15 de setembro de 1965, e revoga as Leis n.s 6.535, de 15 de junho de 1978 e 7.511 , de 7 de julho de 1986). Diário Oficial a União, Brasília, DF (1965). Acesso em jan. 2005. Disponível em: http:// legislação.planalto.gov.br/legislação.nsf

BRUGNARO, C. Valor atribuído pela população às matas ciliares da Bacia do Rio Corumbataí, SP. 2000. 146 f. Tese (Doutorado em Economia Aplicada) - Escola Superior de Agricultura "Luiz de Queiroz", Piracicaba, 2000.
FERREIRA, D. A. C.; DIAS, H. C. T. Situação atual da Mata Ciliar do Ribeirão São Bartolomeu em Viçosa, MG. Revista Árvore, v.28, n.4, p.617-623, 2004.

FRANCO, F. S. Sistemas agroflorestais: uma contribuição para a conservação dos recursos naturais na Zona da Mata de Minas Gerais. 2000. 147f. Dissertação (Mestrado em Ciência Florestal) Universidade Federal de Viçosa, Viçosa, MG, 2000.

FRANCO, F. S. et al. Quantificação de erosão em sistemas agroflorestais e convencionais na Zona de Mata de Minas Gerais. Revista Árvore, v.26, n.6, p.751-760, 2002.

GIL, A. C. Métodos e técnicas de pesquisa social. 4. ed. São Paulo: Atlas, 1995. 208p.

INSTITUTO BRASILEIRO DE GEOGRAFIA E ESTATÍSTICA - IBGE. Censo demográfico 2000. Acesso em mar. 2005. Disponível em: http:/ /www.ibge.gov.br/

MAIA, A.G. Valoração de recursos ambientais. 2002. 199 f. Dissertação (Mestrado em Desenvolvimento Econômico, Espaço e Meio Ambiente) - Universidade Estadual de Campinas, Campinas, 2002.

MARTINS, E.C. O turismo como alternativa de desenvolvimento sustentável: o caso de Jericoacara no Ceará. 2002. 180f. Tese (Doutorado em Economia Aplicada) - Escola Superior de Agricultura "Luiz de Queiroz", Piracicaba, 2002.

SEROA DA MOTTA, R. Manual para valoração econômica de recursos ambientais. Brasília: Ministério do Meio Ambiente, 1998. 218p.

SILVA, R. G.; LIMA, J. E. Valoração contingente do parque "Chico Mendes": uma aplicação probabilística do método Referendum com bidding games. Revista de Econonia e Sociologia Rural, v.42, n.4, p.685-708, 2004.

R. Árvore, Viçosa-MG, v.31, n.2, p.347-353, 2007 